\title{
Purinergic 2X7 receptor is involved in adipogenesis and lipid degradation
}

\author{
JING LI $^{1}$, LINXIA GONG ${ }^{2}$ and QIAOLAN XU ${ }^{1}$ \\ ${ }^{1}$ Pediatric Department, Yancheng Third People's Hospital, Yancheng School of Clinical Medicine of \\ Nanjing Medical University, Yancheng, Jiangsu 224000; ${ }^{2}$ Pediatric Department, Nanjing Integrated \\ Traditional Chinese and Western Medicine Hospital, Nanjing, Jiangsu 210024, P.R. China
}

Received March 19, 2021; Accepted July 20, 2021

DOI: $10.3892 /$ etm.2021.11004

\begin{abstract}
Obesity and dyslipidemia are two metabolic syndrome disorders that have serious effects on the health of patients. Purinergic $2 \mathrm{X}$ receptor ligand-gated ion channel 7 (P2X7R) has been reported to play a role in regulating lipid storage and metabolism. However, the role and potential mechanism of P2X7R in adipogenesis and lipid degradation remain unknown. In the present study, a mouse model of obesity was established by feeding mice a high-fat diet, and the 3T3-L1 cell line was used to analyze the function of $\mathrm{P} 2 \mathrm{X} 7 \mathrm{R}$ in vitro. Reverse transcription-quantitative PCR and western blot analyses were performed to detect the expression levels of P2X7R, sterol regulatory element-binding protein 1 (SREBP1) and other associated transcription factors. Bioinformatics analysis was used to predict the potential target gene of P2X7R and a dual luciferase reporter assay was used to confirm this prediction. Oil Red O staining was used to evaluate the adipogenic capacity of preadipocytes. AdipoRed assay, cholesterol assay and a free glycerol reagent were used to measure the expression levels of triglyceride (TGs), total cholesterol (TC) and glycerin, respectively. The results indicated that P2X7R was highly expressed in obese mice and that it was involved in adipogenic differentiation in vitro. SREBP1 enhanced the transcription activities of $\mathrm{P} 2 \mathrm{X} 7 \mathrm{R}$ to promote its expression. Inhibition of $\mathrm{P} 2 \mathrm{X} 7 \mathrm{R}$ significantly reduced the adipogenic capacity of preadipocytes, decreased the expression levels of adipogenesis-associated transcription factors (peroxisome proliferator-activated receptor $\gamma$, CCAAT-enhancer-binding protein $\alpha$ and fatty-acid-binding protein 4), enhanced the expression levels of lipolytic enzymes (adipose triglyceride lipase, phosphorylated hormone-sensitive lipase and monoacylglycerol lipase) and regulated the expression of TG, TC and glycerin in mature 3T3-L1 cells. These effects
\end{abstract}

Correspondence to: Dr Qiaolan Xu, Pediatric Department, Yancheng Third People's Hospital, Yancheng School of Clinical Medicine of Nanjing Medical University, 20 Xindu Road, Yancheng, Jiangsu 224000, P.R. China

E-mail: qiaolanxuu@163.com

Key words: purinergic $2 \mathrm{X} 7$ receptor, adipogenesis, sterol regulatory element-binding protein 1 were reversed by a small interfering RNA targeting Wnt3a. Therefore, the results suggested that $\mathrm{P} 2 \mathrm{X} 7 \mathrm{R}$, the transcription activities of which were regulated by SREBP1, regulated adipogenesis and lipid degradation by targeting SREBP1, indicating its potential effects on obesity-associated metabolism.

\section{Introduction}

White adipose tissue is a specialized organ that can store fat and release lipids in response to various types of signals (1). It has been shown that adipose tissue regulates metabolic homeostasis by secreting adipokines (2). However, the excessive gathering of adipose tissues can lead to metabolic disorders and promote obesity (3). As of 2016, 39\% of adults ( $\geq 18$ years) were characterized as overweight worldwide (4). Moreover, obesity is associated with various other comorbidities, such as hyperglycemia, diabetes, cardiovascular disease, osteoarthritis and cancer (5). Hypertrophy and hyperplasia of adipocytes are the main causes of obesity and can also result in the development of metabolic disorders (6). Therefore, it is important to understand the detailed mechanism of adipogenesis, which may contribute to the prevention and treatment of obesity and its associated metabolic diseases.

Adipogenesis is a pivotal process required for lipid homeostasis, energy balance and obesity (7). A highly organized signaling cascade involving numerous transcription factors, such as peroxisome proliferator-activated receptor (PPAR) $\gamma$, CCAAT-enhancer-binding protein $(\mathrm{C} / \mathrm{EBP}) \alpha$ and $\mathrm{C} / \mathrm{EBP} \beta$, regulate adipocyte differentiation (8). Increased expression levels of PPAR $\gamma$ and $\mathrm{C} / \mathrm{EBP} \alpha$ are associated with lipid droplet formation and induction of sterol regulatory element-binding protein 1 (SREBP1) expression, as well as of markers of adipocyte maturity, including adiponectin, fatty acid binding protein 4 (FABP4/aP2), perilipin A and fatty acid synthase (9). Previously, the Wnt/ $\beta$-catenin signaling pathway has been discovered to act as a molecular switch during adipogenesis (10); adipogenesis can be inhibited by activating this pathway (11). A recent study found that the adipogenesis regulated by the Wnt pathway could be mediated by its effector TCF7L2 (12). However, to the best of our knowledge, the molecular mechanism underlying the association between adipogenesis and the Wnt/ $\beta$-catenin signaling pathway in obesity still needs to be further clarified. Emerging 
evidence has revealed that Wnt signaling is modulated by P2X7R $(13,14)$.

SREBP1 is a transcription factor that is required for adipogenesis, lipid homeostasis and cellular lipogenesis $(15,16)$. In our previous study, it was shown that SREBP1 could bind to the promoter sites of the purinergic receptor $\mathrm{P} 2 \mathrm{X}$ ligand-gated ion channel 7 (P2RX7) based on analysis of data from a database. P2RX7 is a ligand-gated cation channel encoded by the $\mathrm{P} 2 \mathrm{X} 7$ gene, which belongs to the $\mathrm{P} 2 \mathrm{X}$ receptor family (17). $\mathrm{P} 2 \mathrm{X} 7 \mathrm{R}$ is primarily expressed on cells of hematopoietic, epithelial, mesenchymal and neural lineage, and plays major roles in immunity, neoplasia, inflammation and neurological functions (18). In addition, P2X7R is associated with lipid metabolism (19). P2X7R was also discovered to play an extensive role in controlling lipid storage and metabolism in vivo (20). Suppression of CD36 attenuates adipogenesis via the P2X7R pathway in 3T3-L1 cells (21), and Wnt3a has been shown to mitigate acute lung injury by reducing $\mathrm{P} 2 \mathrm{X} 7$ receptor-mediated alveolar epithelial type I cell death (13). Therefore, it was hypothesized that there may be an association between P2X7R, Wnt signaling and adipogenesis. In the present study, the effects of P2X7R on 3T3-L1 preadipocyte proliferation and differentiation were examined in vitro and in vivo, and the associated underlying mechanism of action was also assessed.

\section{Materials and methods}

Experimental animals. Male 8-week-old C57BL/6J mice $(\mathrm{n}=10$, weight: $19-20 \mathrm{~g}$ ) were obtained from the Animal Core Facility and housed at $22 \pm 2^{\circ} \mathrm{C}$ with a relative humidity of $50 \pm 10 \%$ on a 12 -h light/dark cycle, with free access to food and water. Following 1 week of acclimatization, the mice were randomly assigned into weight-matched groups and fed either a normal chow diet (control; $15 \%$ fat) or a high-fat diet (HFD; 60\% fat; TROPHIC Animal Feed High-Tech Co., Ltd.). The mice were fed a HFD for 12 weeks and blood samples $(0.6 \mathrm{ml})$ were collected from their eyeballs under anesthesia with $2 \%$ pentobarbital sodium $(50 \mathrm{mg} / \mathrm{kg})$. The mice were then sacrificed by cervical dislocation. The fat depots from visceral adipose tissues were immediately removed, frozen in liquid nitrogen and stored at $-80^{\circ} \mathrm{C}$ until required for further analysis. The present study was approved by the Ethics Committee of Yancheng Third People's Hospital (Yancheng, China).

Cell culture and adipocyte differentiation. The 3T3-L1 cell line was purchased from the American Type Culture Collection and cultured in DMEM (Sigma-Aldrich; Merck KGaA) supplemented with $10 \%$ calf serum (Sigma-Aldrich; Merck KGaA). The cells were cultured for 2 days to reach confluence (day 0). Differentiation was induced using DMEM containing $10 \%$ fetal bovine serum (BMG Labtech $\mathrm{GmbH}$ ), $5 \mu \mathrm{g} / \mathrm{ml}$ insulin (Sigma-Aldrich; Merck KGaA), $1 \mu \mathrm{M}$ dexamethasone and $0.5 \mu \mathrm{M}$ 3-isobutyl-1-methylxanthine (Sigma-Aldrich; Merck KGaA) for 2 days. Subsequently, the medium was changed to growth medium (DMEM containing $10 \% \mathrm{FBS}$ and $5 \mu \mathrm{g} / \mathrm{ml}$ insulin), which was used for an additional 2 days (21). The medium was replaced with growth medium and replaced every other day until day 8.
Cell transfection. 3T3-L1 cells $\left(5 \times 10^{4}\right.$ cells/well) were seeded into 6 -well plates $24 \mathrm{~h}$ prior to transfection and cultured at $37^{\circ} \mathrm{C}$ in a humidified incubator with $5 \% \mathrm{CO}_{2}$. Subsequently, the cells were transfected with small interfering RNA (siRNA) targeting SREBP1 (siRNA-SREBP1-1, 5'-GCAAGUCAUUGUUACUAUAAG-3' and 5'-UAUAGU AACAAUGACUUGCUG-3'; siRNA-SREBP1-2, 5'-GAC UGUGUGUCUACA ACUACA-3' and 5'-UAGUUGUAG ACACACAGUCAU-3'; siRNA-SREBP1-NC, 5'-GCAAGU AGCUCUAUUUAUAAG-3' and 5'-UAUAAAAUAGAG CUCUUGCUG-3'), P2X7R (siRNA-P2X7R-1, 5'-GGAACG AUGUCUUGCAGUAUG-3' and 5'-UACUGCAAGACA UCGUUCCAG-3'; siRNA-P2X7R-2, 5'-GCAGUAUGA GACAAACAA AGU-3' and 5'-UUUGUUUGUCUCAUA CUGCAA-3'; siRNA-P2X7-NC, 5'-GGUGUAACGAGU AUCAGUCUG-3' and 5'-GACUGAUACUCGUUACAC CAG-3'), or Wnt3a (siRNA-Wnt3a-1, 5'-GGUCAGCACAUG ACACUAAUG-3' and 5'-UUAGUGUCAUGUGCUGAC CCG-3' and siRNA-Wnt3a-2, 5'-GCUUUAAUCUAGCUG ACAAGA-3' and 5'-UUGUCAGCUAGAUUAAAGCUG -3'; siRNA-Wnt3a-NC, 5'-CGGUCAGCCAACAUACUA GAUG-3' and 5'-UCUAGUAUGUUGUGCUGACCCG-3') using Lipofectamine ${ }^{\circledR} 3000$ (Invitrogen; Thermo Fisher Scientific, Inc.), according to the manufacturer's protocol. Per well, 50 pmol siRNAs were used. The siRNAs were designed and purchased from Shanghai GenePharma Co., Ltd. The cells in the blank control group were untransfected. Following incubation for $48 \mathrm{~h}$ at $37^{\circ} \mathrm{C}$, cells were used for subsequent experiments. Transfection efficiency was assessed using reverse transcription-quantitative PCR (RT-qPCR).

$R T-q P C R$. Total RNA from cells was extracted using TRIzol ${ }^{\circledR}$ reagent (Invitrogen; Thermo Fisher Scientific, Inc.) according to the manufacturer's instructions. Total RNA was reverse transcribed into cDNA using a PrimeScript RT Reagent kit according to the manufacturer's protocol (Invitrogen; Thermo Fisher Scientific, Inc.). qPCR was subsequently performed using SYBR-Green Premix Ex Taq II (Takara Bio, Inc.), following the manufacturer's instructions. GAPDH was used as the internal control. The primer sequences used were as follows: SREBP1 forward, 5'-ACAGTGACTTCCCTG GCCTAT-3' and reverse, 5'-GCATGGACGGGTACATCT TCAA-3'; P2X7R forward, 5'-CACCGTGCTTACAGGTGC TA-3' and reverse, 5'-CGGTCTTGGGGAACTCCTTC-3'; PPAR $\gamma$ forward, 5'-GGGATCAGCTCCGTGGATCT-3' and reverse, 5'-TGCACTTTGGTACTCTTGAAGTT-3'; C/EBP $\alpha$ forward, 5'-GGCCAATGGCATCCAAAATA-3' and reverse, 5'-CCTTGGCGAATTCTGTGAGC-3'; fatty acid binding protein 4 (FABP4) forward, 5'-GCAGTGTTTCTGTGGCTG ACAC-3' and reverse, 5'-GCCATGCACAGGGTCCA-3'; Wnt3a forward, 5'-AGCTACCCGATCTGGTGGTC-3' and reverse, 5'-CAAACTCGATGTCCTCGCTAC-3'; and GAPDH forward, 5'-TGCACCACCAACTGCTTAGC-3' and reverse, 5'-GGCATGGACTGTGGTCATGAG-3'. qPCR was performed on an ABI Prism 7500 Real-Time PCR Detection system (Applied Biosystems; Thermo Fisher Scientific, Inc.) according to the manufacturer's instructions. The thermocycling conditions were as follows: Initial denaturation for $5 \mathrm{~min}$ at $94^{\circ} \mathrm{C}, 35$ cycles of $94^{\circ} \mathrm{C}$ denaturation for $30 \mathrm{sec}$, $55^{\circ} \mathrm{C}$ annealing for $30 \mathrm{sec}$ and $72^{\circ} \mathrm{C}$ extension for $30 \mathrm{sec}$. 
The $2^{-\Delta \Delta \mathrm{Cq}}$ method (22) was used in each sample for relative quantification.

Western blot analysis. The cells were collected and lysed $48 \mathrm{~h}$ after the aforementioned treatments using RIPA lysis buffer (Beyotime Institute of Biotechnology). The protein concentration was calculated using the BCA method. Equal amounts of proteins (30 $\mu \mathrm{g} /$ lane) were resolved using $10-15 \%$ SDS-gels by SDS-PAGE and subsequently transferred onto PVDF membranes (MilliporeSigma). The membranes were incubated with specific antibodies against the following proteins: SREBP1 (1:1,000; cat. no. ab28481), PPAR $\gamma(1: 1,000$; cat. no. ab272718), C/EBP $\alpha$ (1:1,000; cat. no. ab40764), FABP4 (1:2,000; cat. no. ab92501), adipose triglyceride lipase (ATGL; 1:1,000; cat. no. ab207799), phosphorylated hormone-sensitive lipase (p-HSL; 1:100,000; cat. no. ab109400), monoacylglycerol lipase (MGL; 1:2,000; cat. no. ab77398) and Wnt3a (1:1,000; cat. no. ab219412; all purchased from Abcam), and $\beta$-catenin (1:500; cat. no. 8480) and cyclin D1 $(1: 1,000$; cat. no. 55506; both from Cell Signaling Technology, Inc.) at $4^{\circ} \mathrm{C}$ overnight. The membranes were then washed and incubated with HRP-conjugated secondary antibodies (1:10,000; cat. no. 7074; Cell Signaling Technology, Inc.) the following day at room temperature for $2 \mathrm{~h}$. Signals were visualized using chemiluminescence reagent (MilliporeSigma), and densitometry analysis (ImageJ 1.46r; National Institutes of Health) was performed to measure protein expression.

Measurement of triglyceride (TG), total cholesterol (TC) and glycerin levels. The collected blood was centrifuged at $3,000 \times \mathrm{g}$ at $4^{\circ} \mathrm{C}$ for $15 \mathrm{~min}$ to obtain serum. In serum and/or mature 3T3-L1 cells, TG and TC contents were measured with an AdipoRed assay reagent kit (Lonza Group, Ltd.) or Cholesterol assay kit (Abcam), respectively, in accordance with the manufacturers' instructions. Fluorescence was measured following $10 \mathrm{~min}$ of incubation on a Victor3 plate reader (PerkinElmer, Inc.) with the following settings: Excitation (Ex) 485/Emission (Em) 572 nm (for TG) and Ex538/Em587 (for TC).

Differentiated 3T3-L1 adipocytes were serum-starved for $2 \mathrm{~h}$ and treated with AZD1208 (cat. no. HY-15604; MedChemExpress) or isoproterenol, a known lipolysis inducer, for $3 \mathrm{~h}$. Culture medium was obtained and used to measure glycerol contents with a free glycerol reagent (Sigma-Aldrich; Merck KGaA) according to the manufacturer's instructions. The absorbance was determined at $540 \mathrm{~nm}$ using a microplate reader.

Dual luciferase reporter assay. JASPAR software (jaspar. genereg.net/) was used to predict the promoter region of P2X7R, which was a potential target of SREBP1. To confirm this prediction, dual luciferase reporter assays were performed to investigate the regulatory association between SREBP1 and P2X7R. The promoter sites of P2X7R containing the SREBP1 binding site was cloned into a pmirGLO dual luciferase reporter vectors (Promega Corporation). The reporter vectors containing the P2X7R promoter sites and siRNA-SREBP-1 or siRNA-NC were co-transfected into mature 3T3-L1 cells (8 days old) using Lipofectamine ${ }^{\circledR} 3000$ (Invitrogen; Thermo Fisher Scientific, Inc.). Following 48 h of incubation, the cells were lysed and the relative luciferase activities were detected using a Dual Luciferase Reporter assay system (Promega Corporation). Renilla luciferase was used as an internal control for the normalization of luciferase reporter gene in the experiment.

Oil Red $O$ staining. The mature adipocytes were fixed in $10 \%(\mathrm{v} / \mathrm{v})$ formaldehyde solution for $30 \mathrm{~min}$ at room temperature and the cells were stained with $0.5 \%$ Oil Red $\mathrm{O}$ for $30 \mathrm{~min}$ at room temperature. The samples were washed three times with PBS and the cells were visualized using a light microscope (Olympus Corporation; magnification, $\mathrm{x} 400$ ). Oil red O was extracted with $100 \%$ isopropanol and the concentration was determined by measuring the absorbance at $510 \mathrm{~nm}$.

Statistical analysis. The data are expressed as the mean \pm standard deviation. GraphPad Prism 8.0 software (GraphPad Software, Inc.) was used for all statistical analyses. Data were compared using a one-way ANOVA, followed by a Tukey's post hoc test. Each experiment was repeated at least three times. A unpaired Student's t-test was used to compare differences between two groups. $\mathrm{P}<0.05$ was considered to indicate a statistically significant difference.

\section{Results}

$P 2 X 7 R$ is highly expressed in obese mice. The expression levels of P2X7R were determined in visceral adipose tissues of obese mice. C57BL/6J mice were randomly separated into two groups according to their weight. The HFD group was fed a HFD and the control group was fed a normal chow diet for 12 weeks. Mice in the HFD group had significantly increased body weights (52\%) compared with the control group (Fig. 1A). The TG and TC levels in serum were also increased compared with those of the control group (Fig. 1B and C). Western blotting and RT-qPCR analyses indicated that the protein and mRNA levels of P2X7R was increased in the HFD group (Fig. 1D and E). Therefore, the data indicated that P2X7R was highly expressed in obese mice.

$P 2 X 7 R$ is highly expressed during adipogenic differentiation. Subsequently, the specific function of P2X7R during adipogenesis was investigated by assessing $\mathrm{P} 2 \mathrm{X} 7 \mathrm{R}$ expression at different time points during the differentiation of 3T3-L1 cells into mature adipocytes. The P2X7R protein and mRNA levels were increased following adipogenic induction and maintained at a high level of expression during adipocyte differentiation (Fig. 2A and B). In addition, the expression levels of the key transcription factor PPAR $\gamma$, and of the SREBP1 gene, which have been used as adipogenic markers (23-25) are involved in lipogenesis, were assessed. Their expression levels were significantly increased during adipogenic differentiation (Fig. 2C and D). Mature 3T3-L1 cells were cultured for 8 days and used to perform further analysis.

$P 2 X 7 R$ expression levels are reduced following knockdown of SREBPI expression. To investigate the potential molecular mechanism of P2X7R, its putative target genes were screened using bioinformatics analysis. The data indicated that SREBP1, which is a lipid droplet regulator, could bind to the promoter 

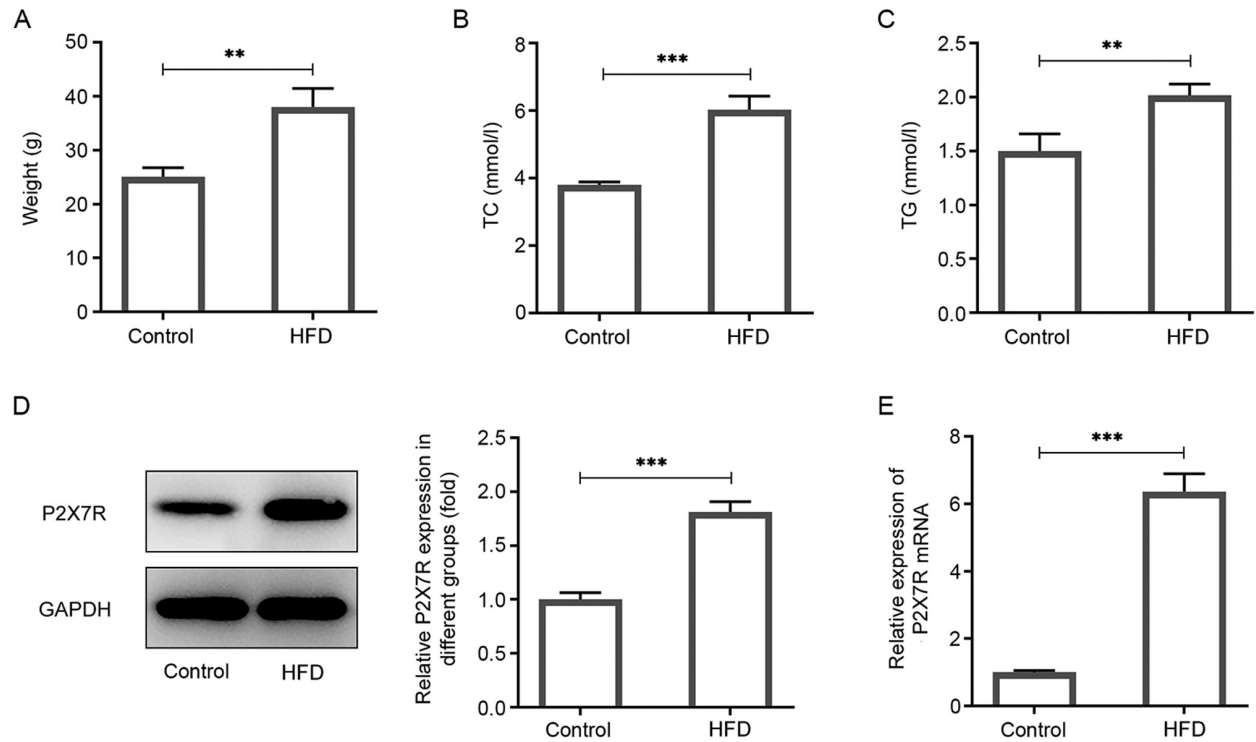

Figure 1. HFD increases the levels of P2X7R. (A) The weight of mice increased in the mice fed a HFD. (B) TC and (C) TG levels in the serum of mice administrated a HFD. P2X7R (D) protein (E) and mRNA levels in visceral adipose tissues of mice fed a HFD or normal diet. Data are presented as the mean \pm standard deviation of five repeats. ${ }^{* *} \mathrm{P}<0.01,{ }^{* * *} \mathrm{P}<0.001$. HFD, high fat diet; TC, total cholesterol; TG, triglyceride; P2X7R, purinergic receptor P2X ligand-gated ion channel 7.

A
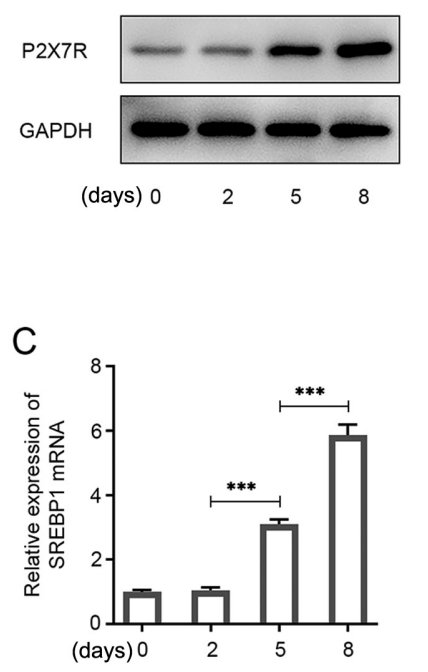

$\mathrm{D}$

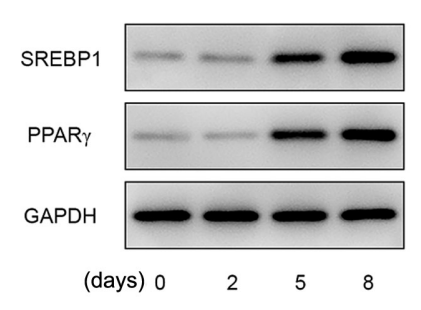

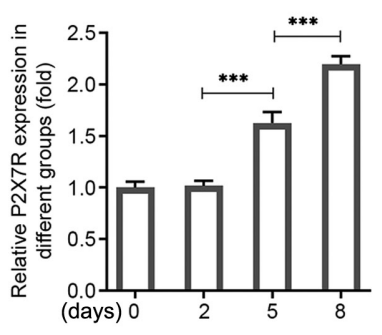

B

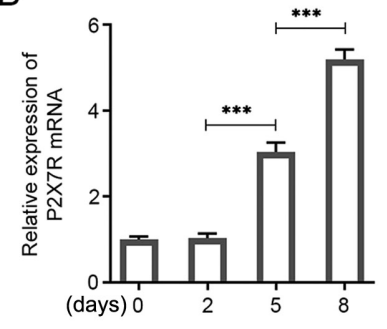

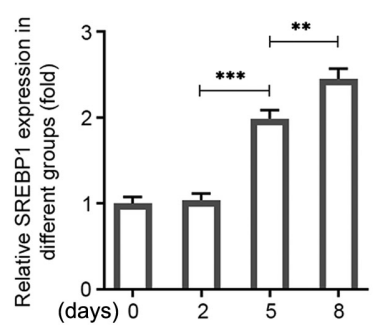

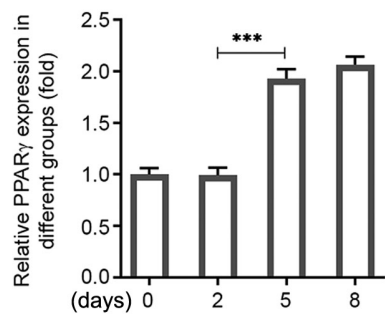

Figure 2. P2X7R expression is significantly upregulated in 3T3-L1 cells during differentiation. P2X7R (A) protein and (B) mRNA levels in the differentiated 3T3-L1 cells. SREBP1 and PPAR $\gamma(C)$ mRNA and (D) protein expression levels in the differentiated 3T3-L1. Data are presented as the mean \pm standard deviation. ${ }^{* *} \mathrm{P}<0.01,{ }^{* * *} \mathrm{P}<0.001$. P2X7R, purinergic receptor P2X ligand-gated ion channel 7; SREBP1; sterol regulatory element-binding protein 1; $\operatorname{PPAR} \gamma$, peroxisome proliferator-activated receptor $\gamma$.

sites of P2X7R. To identify whether SREBP1 participates in the adipogenic process, its expression was knocked down by transfecting siRNA-SREBP1 into mature 3T3-L1 cells following the induction of differentiation. SREBP1 expression was significantly decreased following the transfection of the cells with siRNA-SREBP1-1 and siRNA-SREBP1-2 
A

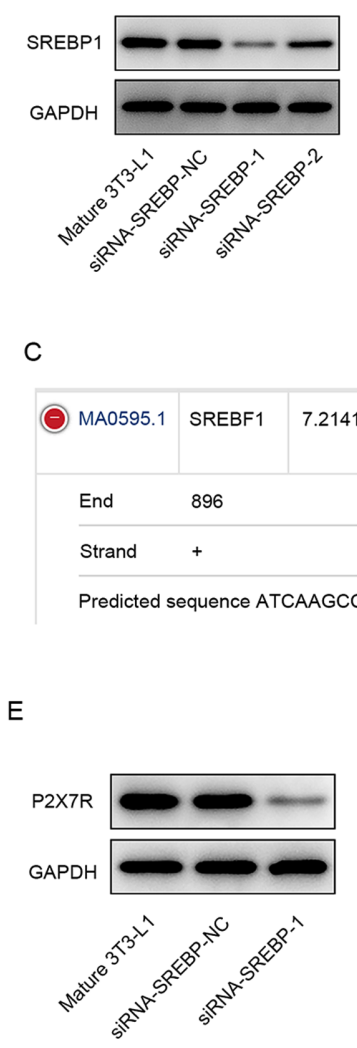

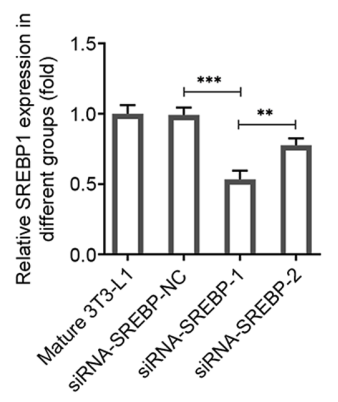

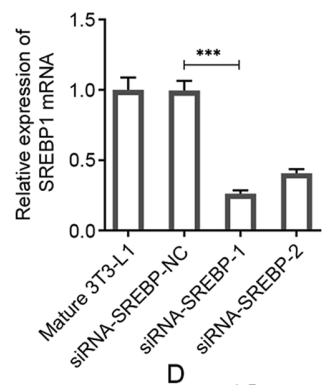

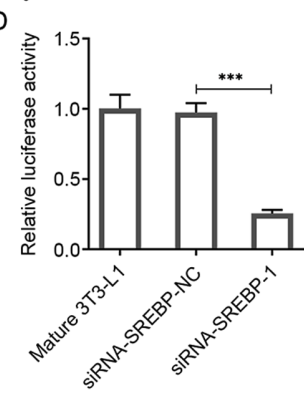

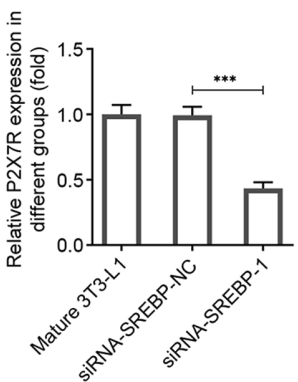

$\mathrm{F}$

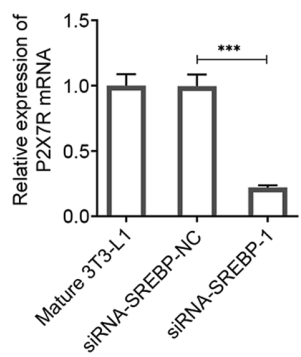

Figure 3. SREBP1 knockdown induces a decrease in the expression of P2X7R. (A) Protein and (B) mRNA expression levels of SREBP-1 following siRNA-mediated knockdown. (C) Putative sites of SREBP-1 binding to the P2X7R promoter region. (D) Luciferase reporter assay for determination of binding of SREPB-1 and P2X7R. P2X7R (E) protein and (F) mRNA levels following knockdown of SREBP-1. Data are presented as the mean \pm standard deviation. ${ }^{* *} \mathrm{P}<0.01$, ${ }^{* * *} \mathrm{P}<0.001$. P2X7R, purinergic receptor P2X ligand-gated ion channel 7; SREBP1; sterol regulatory element-binding protein 1; siRNA small interfering RNA; $\mathrm{NC}$, negative control.

compared with that of mature 3T3-L1 cells, as determined by western blotting and RT-qPCR analyses (Fig. 3A and B). The siRNA-SREBP1-1 group had the lowest expression and was used in subsequent experiments (Fig. 3A and B).

The JASPAR database predicted the binding of SREBP-1 to the P2X7R promoter (Fig. 3C). Subsequently, dual luciferase reporter assays were performed to confirm this prediction. The results indicated that siRNA-SREBP1 significantly inhibited the transcriptional activity of the $\mathrm{P} 2 \mathrm{X} 7 \mathrm{R}$ promoter, whereas the negative control of SREBP1 (siRNA-NC) abolished the inhibitory effect of P2X7R (Fig. 3D). Moreover, P2X7R expression was significantly decreased following transfection of siRNA-SREBP1 into 3T3-L1 cells compared with the corresponding expression noted in the control group (Fig. 3E and F). Overall, these data demonstrated an interaction between P2X7R and SREBP1 and indicated that $\mathrm{P} 2 \mathrm{X} 7 \mathrm{R}$ expression was reduced following knockdown of SREBP1.

Suppression of $P 2 X 7 R$ reduces adipogenic differentiation and lipid accumulation. It has been shown that lipid deposition is associated with the proliferation of preadipocytes and their differentiation into adipose tissue cells (26). To demonstrate the function of P2X7R in adipogenesis, its ability to affect the differentiation of 3T3-L1 preadipocytes was assessed following transfection of the cells with siRNA-P2X7R or siRNA-NC. P2X7R expression was significantly decreased following transfection of 3T3-L1 cells with siRNA-P2X7R-1 and siRNA-P2X7R-2 compared with that observed in the control group as determined by western blotting and RT-qPCR analyses (Fig. 4A and B). The siRNA-P2X7R-1 group exhibited the lowest expression and was used for subsequent experiments. In addition, it was confirmed by Oil Red $\mathrm{O}$ staining that knockdown of P2X7R expression reduced the adipogenic capacity of preadipocytes (Fig. 4C). The AdipoRed assay indicated a reduction in TG accumulation in the siRNA-P2X7R-1 group (Fig. 4D). Collectively, the data revealed that P2X7R promoted 3T3-L1 preadipocyte differentiation.

To determine the effects of P2X7R on lipid accumulation, RT-qPCR and western blot analyses were performed. The adipogenic process is tightly controlled by PPAR $\gamma, \mathrm{C} / \mathrm{EBP} \alpha$ and FABP4 $(27,28)$. In the present study, the expression levels of PPAR $\gamma, \mathrm{C} / \mathrm{EBP} \alpha$ and FABP4 were significantly decreased following transfection of the cells with siRNA-P2X7R-1 compared with the mature 3T3-L1 group (Fig. 5A and B). In addition, lipolysis involves the sequential activity of lipolytic enzymes (ATGL, p-HSL and MGL) (29). The expression levels of ATGL, p-HSL and MGL were significantly increased following transfection of the cells with siRNA-P2X7R-1 compared with that observed in the mature 3T3-L1 cells (Fig. 5C). Moreover, glycerol levels were increased 3-fold in mature 3T3-L1 cells following transfection 
A

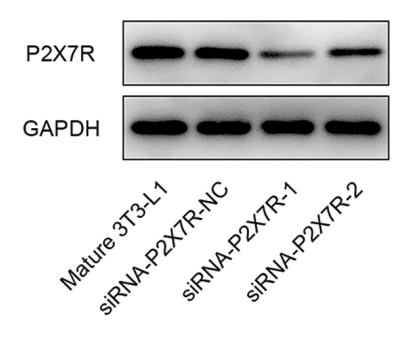

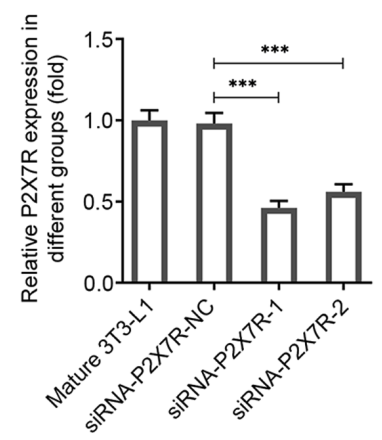

C

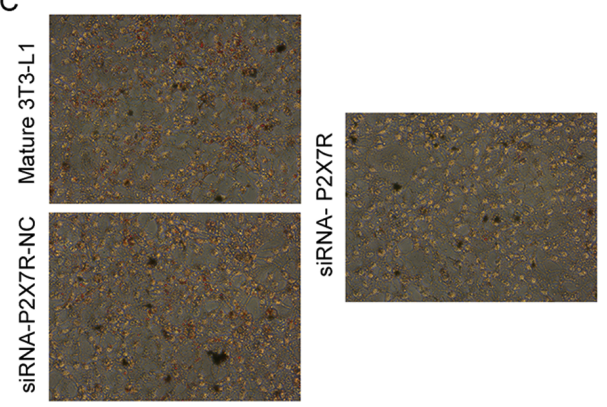

B

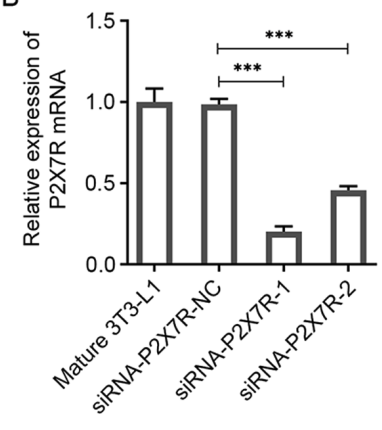

D
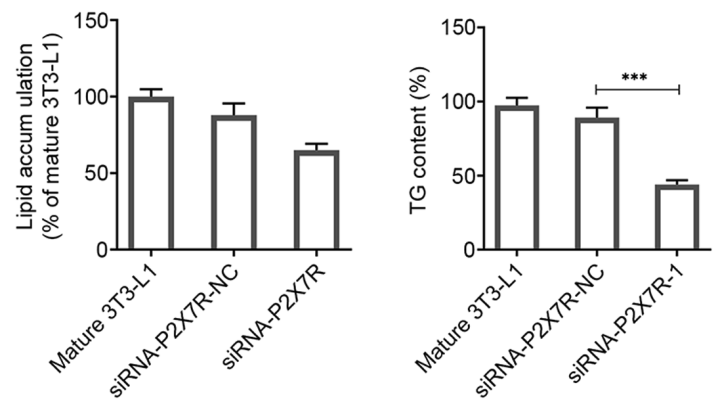

Figure 4. P2X7R knockdown reduces the formation of lipid droplets. P2X7R (A) protein and (B) mRNA expression levels of P2X7R following siRNA-mediated knockdown. (C) Results of Oil red O staining and (D) the TG content following siRNA-mediated knockdown of P2X7R. Data are presented as the mean \pm standard deviation. ${ }^{* * *} \mathrm{P}<0.001$. P2X7R, purinergic receptor P2X ligand-gated ion channel 7; siRNA small interfering RNA; NC, negative control; TG, triglyceride.

with siRNA-P2X7R-1 compared with the control cell group (Fig. 5D). Therefore, the data indicated that P2X7R enhanced adipogenesis and reduced lipolysis in 3T3-L1 cells.

Suppression of $P 2 X 7 R$ reduces adipogenesis. Amongst the factors involved in adipocyte differentiation, Wnt/ $\beta$-catenin has been shown to suppress adipogenesis by inhibiting the expression of PPAR $\gamma$. In order to further explore the mechanism of $\mathrm{P} 2 \mathrm{X7R}$ in the regulation of adipogenesis, the expression levels of the Wnt/ $\beta$-catenin signaling pathway-associated proteins were examined by western blotting following P2X7R interference. Silencing of P2X7R expression significantly promoted the expression levels of Wnt3a, $\beta$-catenin and cyclin D1 (Fig. 6). Next, to investigate the role of wnt3a in the effects of P2X7R on adipogenesis-related markers and lipolysis-related markers, the downregulation of Wnt3a was induced by the transfection of siRNA-Wnt3a-1 or -2 into mature 3T3-L1 cells. As shown in Fig. 7A and B, the protein and mRNA levels of Wnt3a were significantly reduced by siRNA-Wnt3a-1. Moreover, the expression levels of Wnt3a could be reversed by transfection of siRNA-Wnt3a-1 or siRNA-Wnt3a-2 compared with that observed in the P2X7R interference group (Mature 3T3-L1 + siRNA-P2X7R-1; Fig. 7C and D). The mature 3T3L1 + siRNA-P2X7R-1 + siRNA-Wnt3a-1 group exhibited the lowest P2X7R expression levels and was thus used for subsequent experiments.

During adipogenesis, the expression levels of PPAR $\gamma$, $\mathrm{C} / \mathrm{EBP} \alpha$ and FABP4 were significantly decreased following transfection of the cells with siRNA-P2X7R-1 compared with those of the mature 3T3-L1 group (Fig. 7D-F). These effects could be reversed by addition of siRNA-Wnt3a-1 (Fig. 7D-F). During lipolysis, the protein expression levels of ATGL,
p-HSL and MGL were significantly increased following transfection of the cells with siRNA-P2X7R-1 compared with those observed in the mature 3T3-L1 group. These effects could be reversed by transfection of siRNA-Wnt3a-1 (Fig. 7G). In summary, the results indicated that $\mathrm{P} 2 \mathrm{X} 7 \mathrm{R}$ enhanced adipogenesis and reduced lipolysis in 3T3-L1 cells.

\section{Discussion}

Obesity is a serious public health problem and is considered a risk factor for the development of numerous diseases, such as hypertension, type 2 diabetes, coronary heart disease and cancer (6). In the present study, HFD mice were established and 3T3-L1 cells were induced in order to assess adipocyte differentiation. P2X7R was highly expressed in vivo and in vitro. SREBP1 was confirmed to affect the transcription activities of P2X7R and then regulate its expression. Inhibition of P2X7R significantly reduced the adipogenic capacity of preadipocytes, decreased the expression of adipogenesis-associated transcription factors (PPAR $\gamma, \mathrm{C} / \mathrm{EBP} \alpha$ and FABP4), increased the expression levels of lipolytic enzymes (ATGL, p-HSL and MGL) and regulated the contents of TG, TC and glycerin in mature 3T3-L1 cells. These effects could be reversed by application of siRNA-Wnt3a. The results suggested that P2X7R modulation by SREBP1, regulated adipogenesis and lipid degradation.

P2X7 receptors play an important role in multiple biological functions and are present in various tissues (30). Previous in vitro studies have shown that $\mathrm{P} 2 \mathrm{X} 7$ suppressed adipocyte differentiation marker expression by regulating phospholipase and sphingomyelinase activity levels and consequently the production of bioactive lipid signaling molecules $(31,32)$. 
A

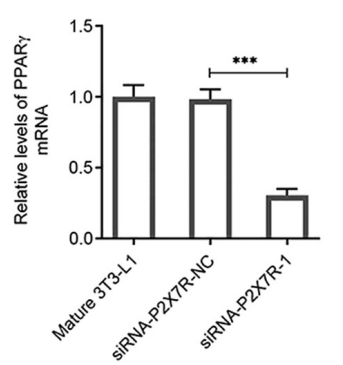

B

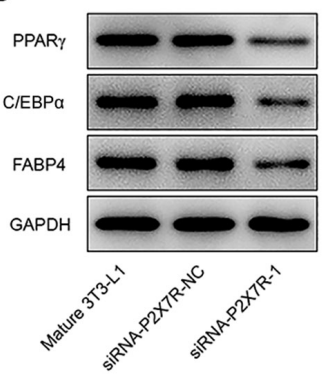

C

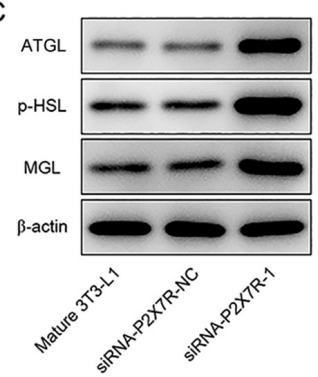

$\mathrm{D}$

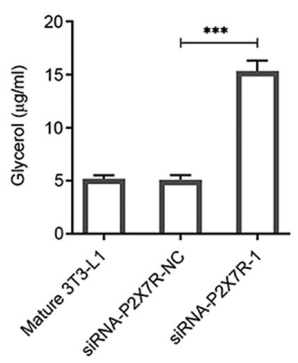

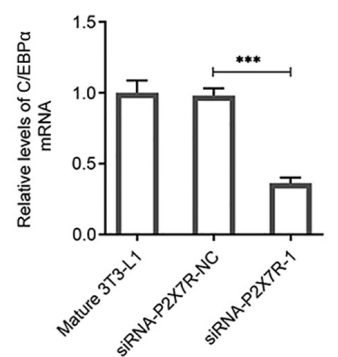
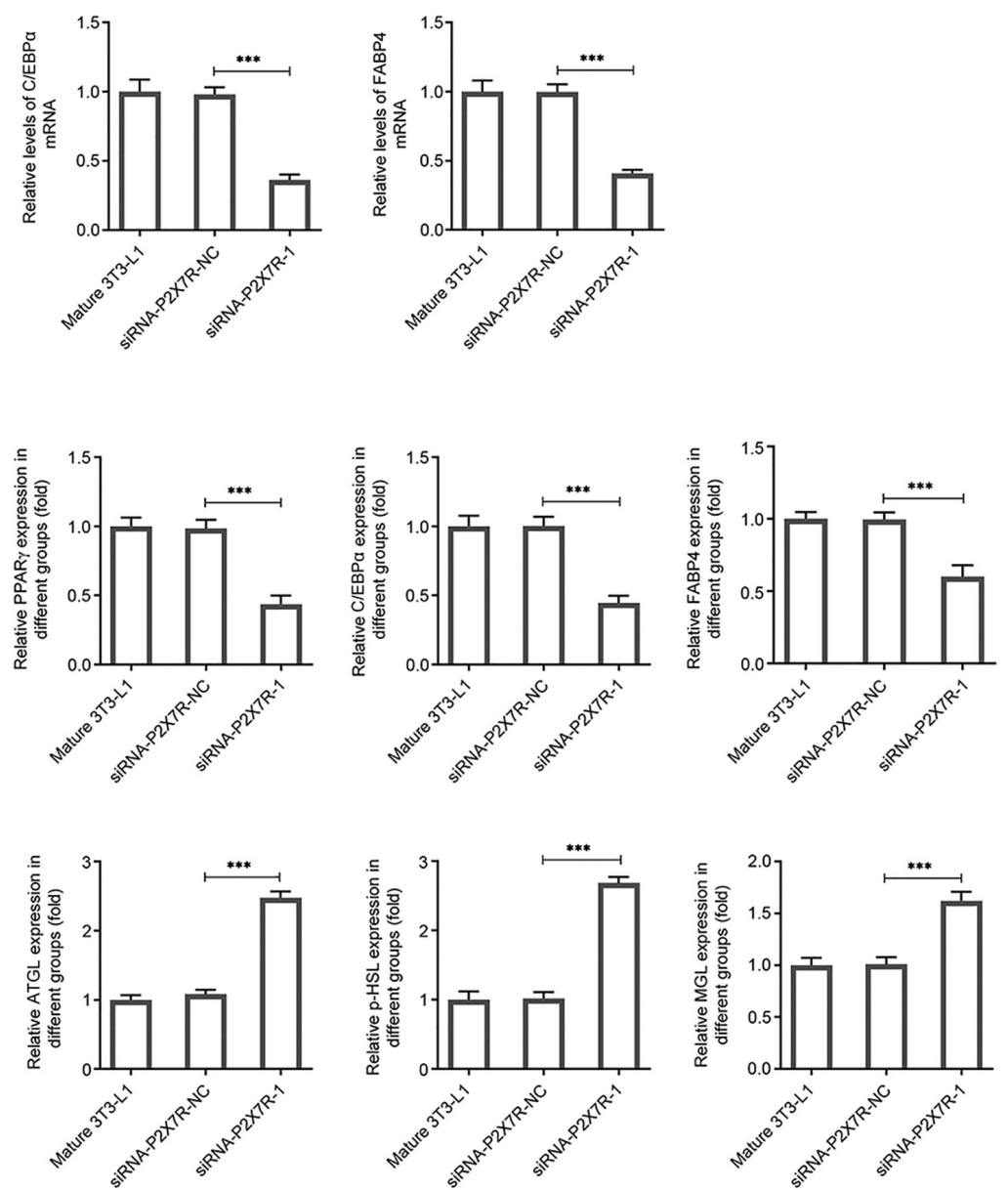

Figure 5. P2X7R knockdown reduces the expression of lipogenesis-related proteins and increases the expression levels of lipolysis-associated enzymes. (A) mRNA and (B) protein expression levels of PPAR $\gamma, \mathrm{C} / \mathrm{EBP} \alpha$ and FABP4 following siRNA-mediated knockdown. (C) Protein expression levels of ATGL, p-HSL and MGL following siRNA-mediated knockdown. (D) Glycerol levels following siRNA-mediated knockdown of P2X7R. Data are presented as the mean \pm standard deviation. ${ }^{* * *} \mathrm{P}<0.001$. P2X7R, purinergic receptor P2X ligand-gated ion channel 7; siRNA small interfering RNA; NC, negative control; PPAR $\gamma$, peroxisome proliferator-activated receptor $\gamma ; \mathrm{C} / \mathrm{EBP} \alpha, \mathrm{CCAAT}-$ enhancer-binding protein $\alpha$; FABP4, fatty acid binding protein 4; ATGL, adipose triglyceride lipase; p-HSL, phosphorylated hormone-sensitive lipase; MGL, monoacylglycerol lipase.
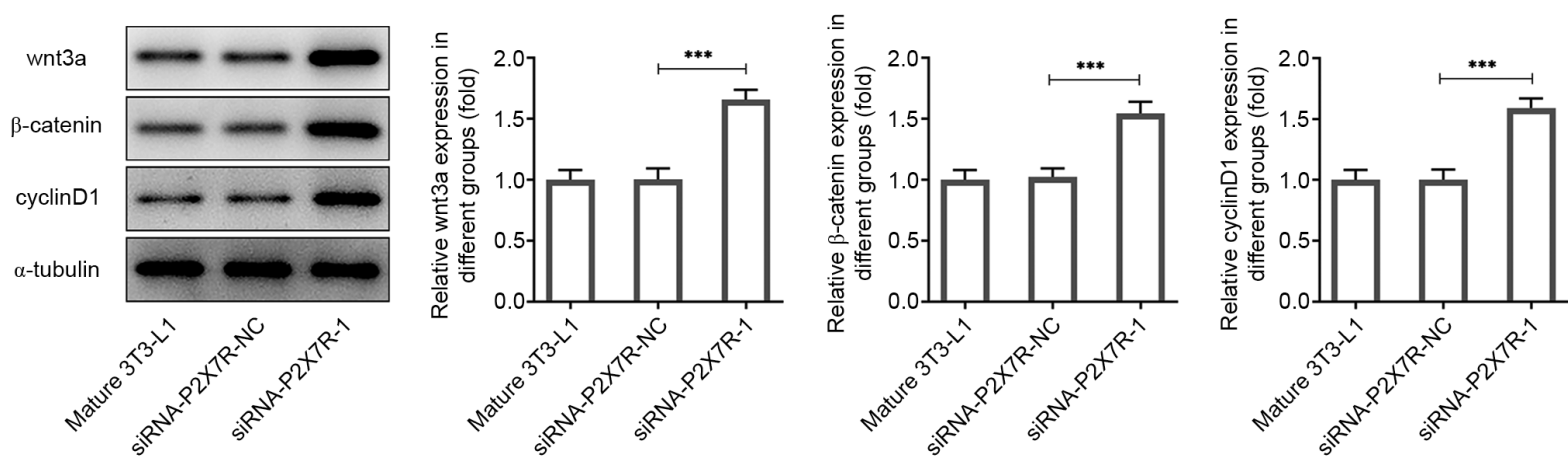

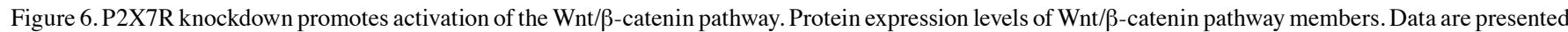
as the mean \pm standard deviation. ${ }^{* * *} \mathrm{P}<0.001$. P2X7R, purinergic receptor P2X ligand-gated ion channel 7; siRNA small interfering RNA; NC, negative control. 
A
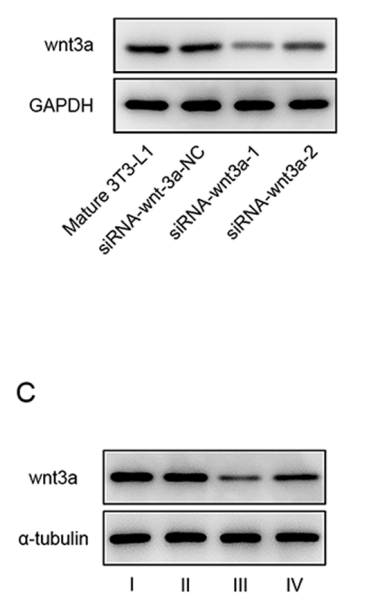

D

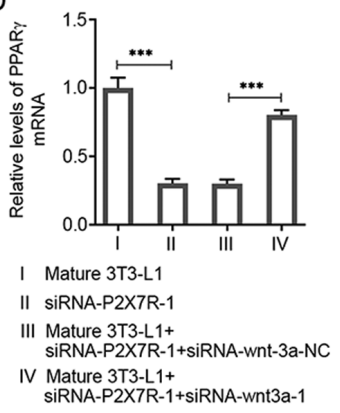

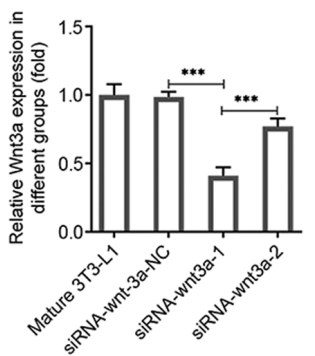

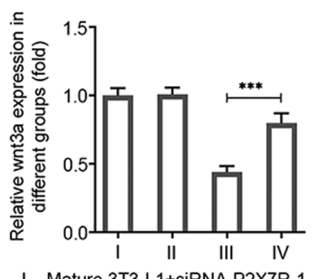

I Mature 3T3-L1+siRNA-P2X7R-1

II Mature 3T3-L1+siRNA-P2X7R-1 +siRNA-wnt-3a-NC

III Mature 3T3-L1+siRNA-P2X7R-1 Mature 3 T3-L1+siRNA-P2X7R-1
+siRNA-wnt3a-2

E

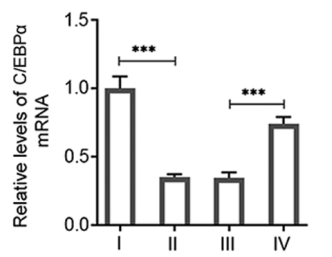

I Mature 3T3-L1

II siRNA-P2X7R-1

III Mature 3T3-L1+

siRNA-P2X7R-1+siRNA-wnt-3a-NC

IV Mature 3T3-L1+

Mature 3 T3-L1+
siRNA-P2X7R-1+siRNA-wnt3a-1
B

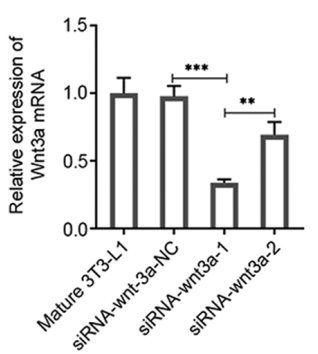

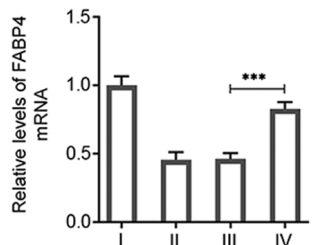

I Mature 3T3-L1

II siRNA-P2X7R-1

III Mature 3T3-L1+

SiRNA-P2X7R-1+siRNA-wnt3a-1

F

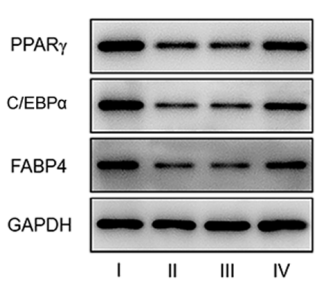

I Mature 3T3-L1

II siRNA-P2X7R-1

III Mature 3T3-L1+

siRNA-P2X7R-1+siRNA-wnt-3a-NC

IV Mature 3T3-L1+
siRNA-P2X7R-1+siRNA-wnt3a-1

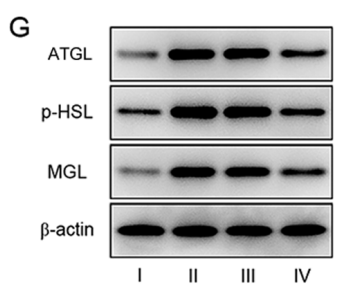

I Mature 3T3-L1
II siRNA-P2X7R-1
III Mature 3T3-L1+
siRNA-P2X7R-1+siRNA-wnt-3a-NC
IV Mature 3T3-L1+
siRNA-P2X7R-1+siRNA-wnt3a-1
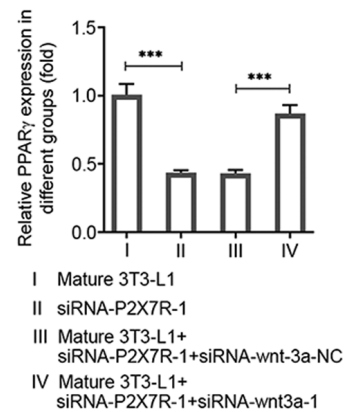

Mature 3T3-L1+
siRNA-P2X7R-1+siRNA-wnt3a-1

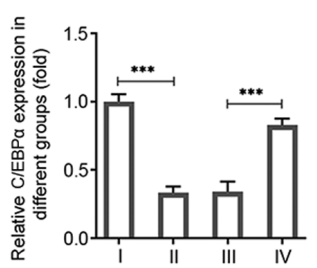

I Mature 3T3-L1
II siRNA-P2X7R-1
III Mature 3T3-L1+
siRNA-P2X7R-1+siRNA-wnt-3a-NC
IV Mature 3T3-L1+
siRNA-P2X7R-1+siRNA-wnt3a-1

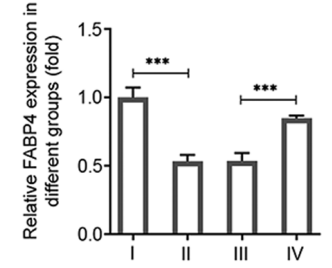

I Mature 3T3-L1

II siRNA-P2X7R-1

III Mature 3T3-L1+

siRNA-P2X7R-1+siRNA-wnt-3a-NC

IV Mature 3T3-L1+

siRNA-P2X7R-1+siRNA-wnt3a-1

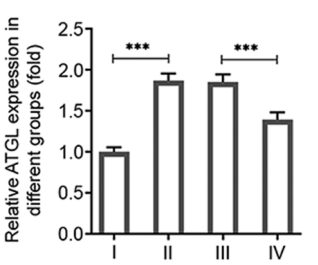

I Mature 3T3-L1

II siRNA-P2X7R-1

III Mature 3T3-L1+

siRNA-P2X7R-1+siRNA-wnt-3a-NC

V Mature 3T3-L1+

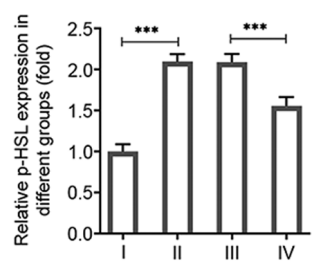

I Mature 3T3-L1
II siRNA-P2X7R-1
III Mature 3T3-L1+
siRNA-P2X7R-1+siRNA-wnt-3a-NC
IV Mature 3T3-L1+
siRNA-P2X7R-1+siRNA-wnt3a-1

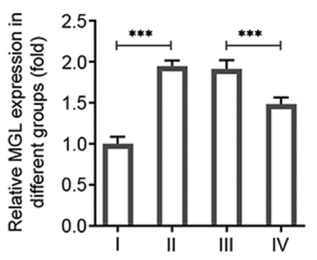

I Mature 3T3-L1

II siRNA-P2X7R-1

III Mature 3T3-L1+

siRNA-P2X7R-1+siRNA-wnt-3a-NC

Mature 3T3-L1+
siRNA-P2X7R-1+siRNA-wnt3a-1

Figure 7.P2X7R regulates the Wnt/ $\beta$-catenin pathway. (A) Protein and (B) mRNA levels of Wnt3a in 3T3-L1 cells following siRNA-wnt3a-1 or siRNA-wnt3a-2 transfection. (C) Protein and (D) mRNA levels of Wnt3a in 3T3-L1 cells following co-transfection of siRNA-P2XR-1 with siRNA-wnt3a-1 or siRNA-wnt3a-2. (E) mRNA and (F) protein expression levels of PPAR $\gamma$, C/EBP $\alpha$ and FABP4. (G) Protein expression levels of ATGL, p-HSL and MGL. Data are presented as the mean \pm standard deviation. ${ }^{* *} \mathrm{P}<0.01,{ }^{* * *} \mathrm{P}<0.001$. $\mathrm{P} 2 \mathrm{X} 7 \mathrm{R}$, purinergic receptor $\mathrm{P} 2 \mathrm{X}$ ligand-gated ion channel 7; siRNA small interfering RNA; NC, negative control; C/EBP $\alpha$, CCAAT-enhancer-binding protein $\alpha$; ATGL, adipose triglyceride lipase; p-HSL, phosphorylated hormone-sensitive lipase; MGL, monoacylglycerol lipase; PPAR $\gamma$, peroxisome proliferator-activated receptor $\gamma$; FABP4, fatty acid binding protein 4 . 
Metabolic syndrome, obesity and type 2 diabetes are relevant risk factors for the increased incidence of chronic kidney disease. The latter is simulated in the laboratory by persistent exposure to HFD (29). In the present study, the HFD mouse model was established and 3T3-L1 cells were induced to differentiate into adipocytes. P2X7R was highly expressed in the HFD mouse model and in mature 3T3-L1 cells. The transcription factors PPAR $\gamma$ and $\mathrm{C} / \mathrm{EBP} \alpha$ are critical for adipogenesis. SREBP1 is another transcription factor, which is required for adipogenesis, lipid homeostasis and cellular lipogenesis $(21,33)$. In adipocytes, the hydrolysis of TGs, which is considered as lipolysis in intracellular lipid droplets, provides stock for fatty acid oxidation. This catabolic process is activated by protein kinase $\mathrm{A}$ and involves various lipolytic enzymes (ATGL, HSL and MGL) (34). A previous study demonstrated that $\mathrm{P} 2 \mathrm{X} 7 \mathrm{R}$ participates in the activation of the NLR family pyrin domain containing 3 inflammasome in podocytes, which may trigger cell damage due to obesity-associated glomerulopathy (17). Loss of P2X7 nucleotide receptor function leads to abnormal fat distribution in mice (20). However, the effects of the elevated expression of P2X7R have not been fully explored. Late adipogenic factors, such as PPAR $\gamma$ and $\mathrm{C} / \mathrm{EBP} \alpha$, are critical transcriptional regulators of lipogenesis (35). A previous study showed that downregulation of PPAR $\gamma, \mathrm{C} / \mathrm{EBP} \alpha$ and FABP4 was involved in the decrease in lipid accumulation (36). Lipolysis is primarily achieved through the gradual hydrolysis of triglycerides via HSL, ATG and MGL to generate free fatty acids (37). Inhibition of P2X7R significantly reduced the adipogenic capacity of preadipocytes, decreased the expression levels of adipogenesis-associated transcription factors (PPAR $\gamma, \mathrm{C} / \mathrm{EBP} \alpha$ and FABP4) and enhanced the expression levels of lipolytic enzymes (ATGL, p-HSL and MGL) (35-37). Therefore, it was concluded that P2X7R was involved in adipogenesis and lipid degradation.

The Wnt family members are evolutionarily conserved secretory lipoproteins, which play an important role in cell proliferation, differentiation and polarity during embryogenesis (38). Previously, the Wnt signaling pathway has been confirmed to play major roles in a series of additional developmental and physiological processes, including adipocyte biology $(11,39)$. However, to the best of our knowledge, the definite function and the underlying mechanism of the Wnt/ $\beta$-catenin signaling pathway in adipogenesis remains unclear. In the present study, silencing of P2X7R expression promoted the expression of Wnt3a, $\beta$-catenin and cyclin D1. The expression levels of Wnt3a were reversed to their initial levels by the addition of siRNA-Wnt3a. It has been previously shown that Wnt signaling prevents the induction of adipogenic regulators, including $\mathrm{C} / \mathrm{EBP} \alpha$ and PPAR $\gamma$ during preadipocyte differentiation. Previously, transient activation of the Wnt/ $\beta$-catenin signaling pathway was shown to inhibit adipogenic regulators in bipotential ST2 cells, which preceded the Wnt-induced increase in osteoblastogenic transcription factors (40). Knockdown of $\mathrm{C} / \mathrm{EBP} \alpha$ and PPAR $\gamma$ is a primary mechanism through which Wnt signaling controls mesenchymal cell fate $(41,42)$. The present study indicated that the expression levels of PPAR $\gamma, \mathrm{C} / \mathrm{EBP} \alpha$ and FABP4 were significantly decreased following transfection with siRNA-P2X7R compared with those noted in the mature 3T3-L1 group. These effects could be reversed by addition of siRNA-Wnt3a.
Moreover, the protein expression levels of ATGL, p-HSL and MGL were significantly increased during lipolysis following transfection of 3T3-L1 cells with siRNA-P2X7R compared with those of the mature 3T3-L1 group. These effects were reversed by addition of siRNA-Wnt3a. Therefore, the data indicated that P2X7R enhanced adipogenesis and reduced lipolysis in 3T3-L1 cells. However, whether this role is related to Wnt/ $\beta$-catenin signaling pathway still requires further studies, and this may be considered a limitation of the present study.

In summary, the present study confirmed that $\mathrm{P} 2 \mathrm{X} 7 \mathrm{R}$ is a novel regulator of 3T3-L1 preadipocyte development. P2X7R promoted 3T3-L1 mature cell adipogenesis and lipid degradation. These findings may enhance the current understanding of P2X7R-regulated adipogenesis and provide a novel model to assess the pathogenesis of obesity.

\section{Acknowledgements}

Not applicable.

\section{Funding}

No funding was received.

\section{Availability of data and materials}

The datasets used and/or analyzed during the current study are available from the corresponding author on reasonable request.

\section{Authors' contributions}

JL and LG conceived and designed the study, and acquired, analyzed and interpreted the data, as well as drafting the manuscript and revising it for important intellectual content. JL, LG and QX performed the experiments and interpreted the data. All authors have read and approved the final manuscript. JL and LG confirm the authenticity of all the raw data.

\section{Ethics approval and consent to participate}

The present study was approved by the Ethics Committee of Yancheng Third People's Hospital (Yancheng, China; approval no. 20200030).

\section{Patient consent for publication}

Not applicable.

\section{Competing interests}

The authors declare that they have no competing interests.

\section{References}

1. Bou M, Todorcevic M, Rodriguez J, Capilla E, Gutierrez J and Navarro I: Interplay of adiponectin, TNF $\alpha$ and insulin on gene expression, glucose uptake and PPARY, AKT and TOR pathways in rainbow trout cultured adipocytes. Gen Comp Endocrinol 205: 218-225, 2014. 
2. Saxton SN, Clark BJ, Withers SB, Eringa EC and Heagerty AM: Mechanistic links between obesity, diabetes, and blood pressure: Role of perivascular adipose tissue. Physiol Rev 99: 1701-1763, 2019.

3. Kahn CR, Wang G and Lee KY: Altered adipose tissue and adipocyte function in the pathogenesis of metabolic syndrome. J Clin Invest 129: 3990-4000, 2019.

4. Bleich SN, Vercammen KA, Zatz LY, Frelier JM, Ebbeling CB and Peeters A: Interventions to prevent global childhood overweight and obesity: A systematic review. Lancet Diabetes Endocrinol 6: 332-346, 2018

5. Bhupathiraju SN and Hu FB: Epidemiology of obesity and diabetes and their cardiovascular complications. Circ Res 118: $1723-1735,2016$

6. Gallagher EJ and LeRoith D: Obesity and diabetes: The increased risk of cancer and cancer-related mortality. Physiol Rev 95: 727-748, 2015.

7. Tang QQ and Lane MD: Adipogenesis: From stem cell to adipocyte. Annu Rev Biochem 81: 715-736, 2012.

8. Haczeyni F, Bell-Anderson KS and Farrell GC: Causes and mechanisms of adipocyte enlargement and adipose expansion. Obes Rev 19: 406-420, 2018

9. Sawamoto A, Nakanishi M, Okuyama S, Furukawa Y and Nakajima M: Heptamethoxyflavone inhibits adipogenesis via enhancing PKA signaling. Eur J Pharmacol 865: 172758, 2019.

10. Im DU, Kim SC, Chau GC and Um SH: Carbamazepine enhances adipogenesis by inhibiting $\mathrm{Wnt} / \beta$-catenin expression. Cells 8 : 1460, 2019.

11. Prestwich TC and Macdougald OA: Wnt/beta-catenin signaling in adipogenesis and metabolism. Curr Opin Cell Biol 19: 612-617, 2007.

12. Chen X, Ayala I, Shannon C, Fourcaudot M, Acharya NK, Jenkinson CP, Heikkinen S and Norton L: The diabetes gene and Wnt pathway effector TCF7L2 regulates adipocyte development and function. Diabetes 67: 554-568, 2018.

13. Guo Y, Mishra A, Weng T, Chintagari NR, Wang Y, Zhao C, Huang $\mathrm{C}$ and Liu L: Wnt3a mitigates acute lung injury by reducing $\mathrm{P} 2 \mathrm{X} 7$ receptor-mediated alveolar epithelial type I cell death. Cell Death Dis 5: e1286, 2014.

14. Sindhavajiva PR, Sastravaha $P$, Arksornnukit $M$ and Pavasant $P$ Purinergic $2 X 7$ receptor activation regulates WNT signaling in human mandibular-derived osteoblasts. Arch Oral Biol 81: 167-174, 2017.

15. Gnoni A, Siculella L, Paglialonga G, Damiano F and Giudetti AM 3,5-diiodo-L-thyronine increases de novo lipogenesis in liver from hypothyroid rats by SREBP-1 and ChREBP-mediated transcriptional mechanisms. IUBMB Life 71: 863-872, 2019.

16. Vadrot N, Duband-Goulet I, Cabet E, Attanda W, Barateau A, Vicart P, Gerbal F, Briand N, Vigouroux C, Oldenburg AR, et al The p.R482W substitution in A-type lamins deregulates SREBP1 activity in Dunnigan-type familial partial lipodystrophy. Hum Mol Genet 24: 2096-2109, 2015.

17. Hou XX, Dong HR, Sun LJ, Yang M, Cheng H and Chen YP: Purinergic $2 X 7$ receptor is involved in the podocyte damage of obesity-related glomerulopathy via activating nucleotide-binding and oligomerization domain-like receptor protein 3 inflammasome. Chin Med J (Engl) 131: 2713-2725, 2018.

18. North RA: Molecular physiology of P2X receptors. Physiol Rev 82: 1013-1067, 2002

19. Su QQ, Tian YY, Liu ZN, Ci LL and Lv XW: Purinergic P2X7 receptor blockade mitigates alcohol-induced steatohepatitis and intestinal injury by regulating MEK1/2-ERK1/2 signaling and egr-1 activity. Int Immunopharmacol 66: 52-61, 2019.

20. Beaucage KL, Xiao A, Pollmann SI, Grol MW, Beach RJ, Holdsworth DW, Sims SM, Darling MR and Dixon SJ: Loss of P2X7 nucleotide receptor function leads to abnormal fat distribution in mice. Purinergic Signal 10: 291-304, 2014.

21. Gao H, Li D, Yang P, Zhao L, Wei L, Chen Y and Ruan XZ: Suppression of CD36 attenuates adipogenesis with a reduction of P2X7 expression in 3T3-L1 cells. Biochem Biophys Res Commun 491: 204-208, 2017.

22. Livak KJ and Schmittgen TD: Analysis of relative gene expression data using real-time quantitative PCR and the 2(-Delta Delta C(T)) method. Methods 25: 402-408, 2001.
23. Han Y, Lee SH, Lee IS and Lee KY: Regulatory effects of 4-methoxychalcone on adipocyte differentiation through PPAR $\gamma$ activation and reverse effect on TNF- $\alpha$ in 3T3-L1 cells. Food Chem Toxicol 106: 17-24, 2017.

24. Lee CW, Seo JY, Lee J, Choi JW, Cho S, Bae JY, Sohng JK, Kim SO, Kim J and Park YI: 3-O-Glucosylation of quercetin enhances inhibitory effects on the adipocyte differentiation and lipogenesis. Biomed Pharmacother 95: 589-598, 2017.

25. Ma J, Liao H, Lin Y, Huang K, Zhu J and Wang Y: Molecular characterization, expression analysis of Chemerin gene and its potential role in intramuscular adipocyte differentiation of goat Anim Biotechnol 31: 382-390, 2020.

26. Rosen ED and MacDougald OA: Adipocyte differentiation from the inside out. Nat Rev Mol Cell Biol 7: 885-896, 2006.

27. Moseti D, Regassa A and Kim WK: Molecular regulation of adipogenesis and potential anti-adipogenic bioactive molecules. Int J Mol Sci 17: 124, 2016.

28. Zhang L, Zhang L, Wang X and Si H: Anti-adipogenic effects and mechanisms of ginsenoside $\operatorname{Rg} 3$ in pre-adipocytes and obese mice. Front Pharmacol 8: 113, 2017.

29. Jin H, Lee K, Chei S, Oh HJ, Lee KP and Lee BY: Ecklonia stolonifera extract suppresses lipid accumulation by promoting lipolysis and adipose browning in high-fat diet-induced obese male mice. Cells 9: 871, 2020.

30. Di Virgilio F, Dal Ben D, Sarti AC, Giuliani AL and Falzoni S: The P2X7 receptor in infection and inflammation. Immunity 47: 15-31, 2017

31. Coccurello R and Volonte C: P2X7 Receptor in the management of energy homeostasis: Implications for obesity, dyslipidemia, and insulin resistance. Front Endocrinol (Lausanne) 11: 199, 2020

32. Calderon-Dominguez M, Mir JF, Fucho R, Weber M, Serra D and Herrero L: Fatty acid metabolism and the basis of brown adipose tissue function. Adipocyte 5: 98-118, 2015.

33. Moon MH, Jeong JK, Lee YJ, Seol JW, Ahn DC, Kim IS and Park SY: $18 \beta$-Glycyrrhetinic acid inhibits adipogenic differentiation and stimulates lipolysis. Biochem Biophys Res Commun 420: 805-810, 2012

34. Seo YJ, Jin H, Lee K, Song JH, Chei S, Oh HJ, Oh JH and Lee BY: Cardamonin suppresses lipogenesis by activating protein kinase A-mediated browning of 3T3-L1 cells. Phytomedicine 65: $153064,2019$.

35. Lee JE, Schmidt H, Lai B and Ge K: Transcriptional and epigenomic regulation of adipogenesis. Mol Cell Biol 39: e00601-e00618, 2019.

36. Walther TC and Farese RV Jr: Lipid droplets and cellular lipid metabolism. Annu Rev Biochem 81: 687-714, 2012.

37. Suh HJ, Cho SY, Kim EY and Choi HS: Blockade of lipid accumulation by silibinin in adipocytes and zebrafish. Chem Biol Interact 227: 53-62, 2015

38. Clevers $\mathrm{H}$ : Wnt/beta-catenin signaling in development and disease. Cell 127: 469-480, 2006.

39. Xi FX, Wei CS, Xu YT, Ma L, He YL, Shi XE, Yang GS and Yu TY: MicroRNA-214-3p targeting Ctnnb1 promotes 3T3-L1 preadipocyte differentiation by interfering with the Wnt//-catenin signaling pathway. Int J Mol Sci 20: 1816, 2019.

40. Kang S, Bennett CN, Gerin I, Rapp LA, Hankenson KD and Macdougald OA: Wnt signaling stimulates osteoblastogenesis of mesenchymal precursors by suppressing CCAAT/enhancer-binding protein alpha and peroxisome proliferator-activated receptor gamma. J Biol Chem 282: 14515-14524, 2007.

41. Liu J and Farmer SR: Regulating the balance between peroxisome proliferator-activated receptor gamma and beta-catenin signaling during adipogenesis. A glycogen synthase kinase 3beta phosphorylation-defective mutant of beta-catenin inhibits expression of a subset of adipogenic genes. J Biol Chem 279: 45020-45027, 2004.

42. Liu J, Wang H, Zuo Y and Farmer SR: Functional interaction between peroxisome proliferator-activated receptor gamma and beta-catenin. Mol Cell Biol 26: 5827-5837, 2006. International (CC BY-NC-ND 4.0) License. 